\title{
Relato de Experiência de Educação Online - Avaliação em IHC Abordando a Finitude da Vida
}

\author{
Jorge Silva ${ }^{1}$, Sílvia Amélia Bim ${ }^{1}$, Daniele Trevisan², Cristiano Maciel ${ }^{2,3}$ \\ ${ }^{1}$ Departamento Acadêmico de Informática - UTFPR \\ Curitiba - PR - Brazil \\ ${ }^{2}$ Instituto de Computação - Universidade Federal de Mato Grosso - UFMT \\ Cuiabá-MT-Brasil
}

${ }^{3}$ Programa de Pós-Graduação em Educação - Universidade de Mato Grosso - UFMT Cuiabá-MT-Brasil

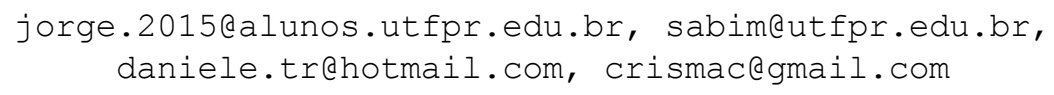

\begin{abstract}
The COVID-19 pandemic imposed limitations to formal classroom education scenarios. On the other hand, these limitations were also inviting to innovation, be it regarding the perspective on educational practices or presented contents. This article presents an experience report from the point of view of an Information Systems student, in the context of the Human-Computer Interaction Evaluation subject. Methods of inspection and observation were employed in the configuration of Facebook's digital legacy tool. This way, the finitude of life subject was analysed, allowing social and communication skills to be developed alongside technical abilities.
\end{abstract}

Resumo. A pandemia de COVID-19 impôs limitações para os cenários de educação formal presencial. Por outro lado, essas limitações também convidaram para a inovação, seja na perspectiva das práticas educacionais ou nos conteúdos abordados. $O$ presente artigo apresenta um relato de experiência, na percepção de um estudante de Sistemas de Informação, no contexto da disciplina de Avaliação em Interação Humano-Computador. Métodos de inspeção e observação foram aplicados na configuração de legado digital do Facebook. Dessa forma, o tema da finitude da vida foi abordado permitindo que as habilidades sociais e comunicativas fossem desenvolvidas, além das habilidades técnicas.

\section{Introdução}

As limitações impostas pela pandemia de COVID-19 exigiram mudanças de comportamento em diferentes contextos, sendo um deles o ambiente de educação formal, como o de cursos superiores. Além disso, a pandemia trouxe a finitude da vida à tona nas diferentes mídias e no cotidiano das pessoas. Diante de tantos desafios, inúmeras estratégias vêm sendo criadas para o contexto educacional [Pimentel and Carvalho, 2020] e muitas oportunidades surgem para inovar não apenas na perspectiva das tecnologias e metodologias utilizadas, mas também no conteúdo abordado. Dessa 
forma, [Trevisan and Maciel, 2020] sugerem que o tema da morte seja discutido no contexto da sala de aula, articulado com os conceitos de tecnologia.

Buscando tornar o processo formativo de estudantes de Sistemas de Informação e Engenharia de Computação mais alinhado com o cenário atual, oferecendo uma formação além dos aspectos técnicos [Oliveira et al., 2020] a disciplina de Avaliação em Interação Humano-Computador (IHC), utilizando Educação OnLine (EOL) como abordagem didático-pedagógica [Pimentel and Carvalho, 2020], incluiu a morte como tema dos trabalhos a serem conduzidos durante o segundo semestre letivo de 2020.

O presente artigo apresenta o relato da experiência vivenciada por um estudante da disciplina, tanto na perspectiva técnica quanto na perspectiva das habilidades sociais desenvolvidas [Oliveira et al., 2020]. Assim, descreve-se a motivação para sua escrita na seção 2. A disciplina, a turma e as atividades realizadas são descritas na seção 3 . Na seção 4 são apresentados os resultados técnicos obtidos pela aplicação de três métodos de Avaliação em Interação Humano-Computador (IHC) na configuração de contato herdeiro do Facebook. As reflexões sobre as habilidades sociais desenvolvidas e as considerações finais sobre a experiência são apresentadas na seção 5.

\section{Motivação}

Segundo [Trevisan and Maciel, 2020], rituais fúnebres são um fator importante no contexto do luto para prestar as últimas homenagens a um falecido. Durante a pandemia de COVID-19, tais rituais se tornaram não recomendados pelo Ministério da Saúde e tiveram de seguir diretrizes reduzindo a quantidade de presentes. Dadas tais limitações, ferramentas digitais - como por exemplo a conta memorial do Facebook - têm sido utilizadas por usuários da internet para contornar o risco de infecção pela COVID-19 em um funeral comum, sem comprometer por completo o processo de luto. Os autores destacam ainda a necessidade de abordar o tema da morte no contexto da educação para outras áreas além da filosofia, tais como disciplinas tecnológicas que envolvam computação e sociedade, tendo em vista que dados e informações não morrem junto com seus autores, e podem ser destinados a construção de memórias e legados digitais.

Em 2019, a rede social Facebook disponibilizou oficialmente para todos os usuários uma configuração para definição de conta de herdeiro [Taylor, 2019]. O objetivo é dar ao usuário uma oportunidade de repassar seus bens digitais para alguém de confiança após seu falecimento, ou de transformar sua conta em um memorial. Também permite alternativamente a exclusão da conta. Caso a configuração não tenha sido definida antes do falecimento, um familiar pode solicitar via formulário os direitos para gerenciamento da conta memorial ou exclusão do perfil. [Viana et al., 2017] realizam uma análise e comparação das ações de legado digital disponibilizadas por algumas das redes sociais mais utilizadas em 2017, incluindo o Facebook.

Considerando a necessidade de abordar o tema da morte no contexto educacional [Trevisan and Maciel, 2020] e o uso crescente do Facebook para rituais fúnebres, identificou-se a oportunidade de avaliar o Facebook, como descrito em [Viana et al., 2017], no contexto da disciplina de Avaliação em IHC. 


\section{Descrição da Experiência}

A experiência relatada foi vivenciada no contexto da disciplina Avaliação em IHC da Universidade Tecnológica Federal do Paraná, campus Curitiba-PR, ofertada para os cursos de Sistemas de Informação e Engenharia de Computação, com carga horária de 45h/aula. Trata-se de uma disciplina optativa que compõe a trilha de IHC e tem como pré-requisito a disciplina de Introdução à IHC. Face às limitações da pandemia, a disciplina foi realizada de modo totalmente online durante 23 de fevereiro a 25 de maio de 2021, referente ao semestre letivo de 2020/2. Diversas ferramentas computacionais foram utilizadas: Google Meet para os encontros síncronos semanais de 2 horas de duração, Google Classroom para disponibilização de materiais e entrega de tarefas assíncronas e Telegram para atendimento assíncrono durante a semana.

A turma, com 7 estudantes, foi dividida em dois grupos para realização das tarefas em dois sistemas distintos: YouTube e Facebook. Cada estudante escolheu o sistema computacional interativo a ser avaliado por afinidade (conhecimento e uso) e assim os grupos foram formados.

O tema da morte foi apresentado no primeiro encontro síncrono da disciplina junto com a apresentação do plano de ensino. Textos sobre legado digital foram indicados como leitura para discussão no segundo encontro síncrono. O conteúdo da disciplina contempla: personas, questionários, métodos por inspeção - Avaliação Heurística (AH) e Método de Inspeção Semiótica (MIS) e um método por observação Método de Avaliação de Comunicabilidade (MAC). Todos os conteúdos foram explorados com foco em legado digital nos sistemas indicados pela docente.

Nesse artigo estão descritos os resultados e a experiência de um estudante que aplicou os métodos na avaliação da configuração do legado digital do Facebook. O estudante está no quarto período do curso de Sistemas de Informação e antes de Avaliação em IHC havia cursado as disciplinas de IHC (pré-requisito) e Acessibilidade e Inclusão Digital. Além disso, é técnico em informática e desenvolvedor full-stack com cinco anos de experiência na área, atualmente trabalhando em uma empresa que vende sistemas para instituições religiosas de várias vertentes. Com relação aos métodos de avaliação em IHC, tinha a experiência prévia de aplicação do método no contexto da disciplina de Acessibilidade e Inclusão Digital e com relação ao tema da finitude da vida articulado com aspectos técnicos a experiência na disciplina de Avaliação em IHC foi a primeira.

\section{Resultados das Avaliações de IHC}

Nesta seção estão descritos os resultados da aplicação de três métodos de avaliação de IHC: AH, MIS e MAC na configuração do legado digital do Facebook.

Inicialmente, cada estudante criou uma persona [Barbosa et al., 2021]. A persona que guiou as avaliações desse relato de experiência foi: Pedro Something, 28 anos. Não possui religião e sua crença é de que a morte é o fim total de sua existência, não acreditando no pós-vida nem reencarnação. Pedro tem interesse de que sua conta seja repassada a alguém de confiança caso se torne permanentemente incapacitado de acessá-la novamente. 
Em seguida, foi elaborado um cenário de inspeção, uma história criada pela equipe de desenvolvimento para descrever um possível caso de uso para uma pessoa [Barbosa et al., 2021]: Pedro, a convite de um amigo, atendeu a uma palestra sobre legado digital. Seu amigo perdeu recentemente um ente querido e passou a ter preocupação com o legado deixado. Após ter uma introdução ao assunto, Pedro se interessou por verificar como proceder para configurar a ferramenta de configuração de contato herdeiro para sua conta do Facebook.

O framework DECIDE [Preece et al., 2013] foi instanciado para conduzir as avaliações, realizadas em um notebook com sistema operacional Windows 10, e a interface foi modificada de acordo com uma configuração do Facebook que torna os textos em auto contraste, denominada "Tema Escuro".

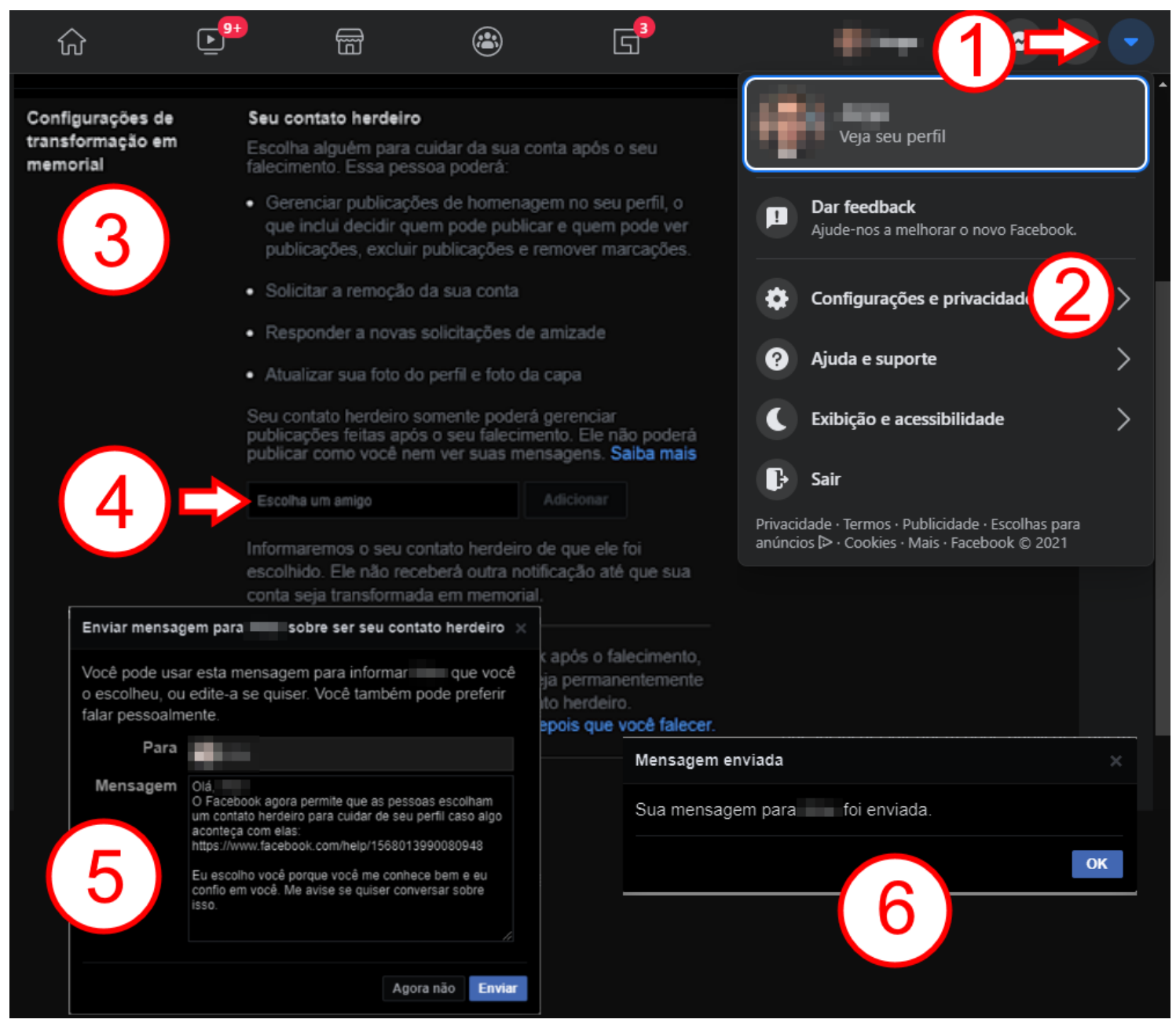

Figura 1. Representação visual dos passos e telas por onde o usuário deve percorrer para configuração. Capturas de tela obtidas em 11/04/2021

O processo de configuração de contato herdeiro pode ser realizado através dos seguintes passos, conforme ilustra a Figura 1:

1. Acessar o menu localizado no canto superior direito da tela.

2. Clicar em "configurações e privacidade" após clicar no menu. 
3. Na primeira tela é possível identificar a configuração de contato herdeiro dentre um de vários itens de privacidade sendo listados. Clicá-lo irá exibir mais informações e opções de configuração.

4. Em um campo de texto dentro da tela mencionada, buscar e clicar em um contato de confiança no Facebook.

5. Será aberta uma tela permitindo enviar uma mensagem à pessoa escolhida informando que em caso de falecimento, ele passa a ter o direito de gerenciar a conta. A mensagem é configurável e vem preenchida com um texto padrão.

6. Confirmação de envio de mensagem ao clicar em "Enviar".

$\mathrm{Na} \mathrm{AH}$, a inspeção individual realizada pelo estudante desse relato de experiência, indicou a violação de 3 heurísticas: Flexibilidade e eficiência de uso no Menu para acesso da tela de configurações (severidade 2, problema pequeno), Projeto estético e minimalista na Tela de configuração de contato herdeiro (severidade 1, problema estético) e Ajuda e documentação na Confirmação de envio de mensagem e conclusão de configuração (severidade 2 , problema pequeno).

Durante a $\mathrm{AH}$, foram observados os pontos descritos a seguir, que não puderam ser classificados pelas heurísticas gerais de usabilidade [Nielsen, 1994]: (1) Linguagem não compassiva A palavra falecimento é exibida três vezes na tela. Embora seja uma alternativa mais suave para a palavra "morte", essa palavra pode causar desconforto, considerando que o contexto do texto envolve o próprio indivíduo, impactando no tempo para realizar a tarefa de configuração ou no abandono da mesma. Considerando que, de acordo com [Trevisan and Maciel, 2020], o tema é tabu na sociedade brasileira, e considerando a estratégia do YouTube, cuja ferramenta de legado digital também foi avaliada como atividade inicial da disciplina, e que não utiliza a palavra falecimento e nem a palavra morte, a palavra falecimento poderia ser evitada; (2) Violação de privacidade $\mathrm{O}$ contato escolhido como herdeiro é imediatamente avisado de que o usuário lhe indicou para administrar a conta após clicar em Enviar (destaque 5 da Figura 1). Pode ocorrer, por exemplo, do usuário estar apenas explorando a ferramenta e não ter avisado o amigo escolhido. $\mathrm{O}$ envio prematuro da mensagem pode causar ansiedade na pessoa herdeira e/ou impactar nas relações sociais dos envolvidos. Seria interessante permitir que o usuário escolha quando o herdeiro será avisado.

O MIS [de Souza et al., 2006] foi aplicado individualmente e gerou uma instância da metamensagem, consolidando as inspeções de signos metalinguísticos, estáticos e dinâmicos, indicando que as metamensagens segmentadas são complementares, oferecendo uma boa comunicabilidade para a tarefa de configuração do contato herdeiro. A metamensagem consolidada é apresentada a seguir.

O que este usuário quer fazer, de que formas preferenciais e por quê? Escolher uma pessoa para cuidar da sua conta de Facebook após o seu falecimento. Essa pessoa será alguém com quem o usuário já tem uma relação de amizade no Facebook. Ou seja, a pessoa também precisa ter uma conta nessa rede social. Após escolher o herdeiro o usuário quer enviar uma mensagem avisando sobre a sua decisão. Que sistema o designer construiu para este usuário (visão de design)? Na visão de design, cuidar da conta de alguém após o seu falecimento consiste em ter permissão para algumas tarefas específicas (destaque 3 da Figura 1). Ao decidir sobre quem herdará a conta, o usuário deverá enviar uma mensagem comunicando sobre essa decisão. A mensagem será 
enviada imediatamente para a pessoa escolhida. Como e para que o usuário deve se comunicar com o sistema (como funciona)? O usuário não pode configurar o que exatamente o herdeiro poderá fazer. Há um conjunto fixo de tarefas que o herdeiro poderá realizar e isso não pode ser alterado. O herdeiro será avisado duas vezes: assim que a mensagem de notificação for enviada e quando a conta se tornar um memorial. Sobre a mensagem de notificação há três possibilidades: (a) possibilidade de enviar o texto pré-configurado na caixa de escrita; ou (b) a possibilidade de alteração do texto pré-configurado na caixa de escrita; ou (c) a possibilidade de informar o contato herdeiro pessoalmente.

Complementando o MIS, com relação ao design do Tema Escuro, o contraste entre as fontes e o fundo pode prejudicar a legibilidade da tela se o usuário estiver em um ambiente com pouca luminosidade. A palavra falecimento aparece três vezes na interface principal e isso pode gerar desconforto no usuário.

A avaliação por observação foi realizada com apenas uma participante, considerando-se a limitação de tempo da disciplina. Embora os métodos recomendem um número maior de participantes, considera-se que a observação feita com apenas um participante e um observador seja um exercício didático que promove o conhecimento e a reflexão sobre as possibilidades do método. A participante é do círculo social do avaliador, com conhecimento na rede social Facebook compatível com o da persona, sem conhecimento sobre legado digital.

A observação foi avaliada com relação ao critério de comunicabilidade e identificou-se as seguintes rupturas: Cadê? (Procurando seção da configuração de contato herdeiro na tela de configurações) E agora? (Acessou uma tela errada no menu lateral esquerdo) Socorro (Necessária intervenção verbal com o avaliador para voltar pra tela anterior) (Realização da existência da caixa de texto e sua finalidade) Pra mim está bom (Inseriu o nome mas não clicou em adicionar) Epa (percebeu que faltou clicar em adicionar) E agora? (Enviou a mensagem, não sabe se finalizou) E agora? (Marcou para enviar arquivo de permissão, não sabe se finalizou)

A experiência de uso é confusa em um primeiro acesso mesmo para quem está habituado com o Facebook. Algumas considerações levantadas a partir da observação são: (a) Ter a seção "Configuração de conta de herdeiro" disponível na tela inicial de configurações não é o suficiente para chamar atenção para a configuração. (b) Selecionar um amigo não abre automaticamente a tela de envio de mensagem, a pessoa pode não perceber que precisa clicar em "Adicionar" para continuar a configuração. (c) A pop-up após o envio da mensagem não é clara o suficiente para informar se as configurações mínimas estão finalizadas. Não fica claro se clicar no botão "Fechar", que aparece após enviar a mensagem, abaixo da caixa indicada pelo círculo 4 na Figura 1, fará com que a configuração seja salva ou não.

\section{Considerações}

Segundo a percepção do estudante, o estudo e aplicação de métodos de avaliação em IHC permitiram o exercício de uma comunicação oral e escrita ampla quando comparada a outras disciplinas, tendo em vista a necessidade constante de troca de informações entre estudantes, bem como o recrutamento de participantes para algumas etapas das avaliações. A qualidade e quantidade de informações coletadas nessas 
interações foram primordiais para a escrita de grande parte dos relatórios que deveriam ser entregues como atividades da disciplina. A partir dos pontos analisados através dessas interações, também foi possível perceber as limitações de cada método.

Dentre os três métodos aplicados, a $\mathrm{AH}$ foi a mais rápida para aplicar, porém a que menos revelou informações devido a pequena quantidade de heurísticas violadas, $o$ que restringe demais a quantidade de pontos que podem ser considerados. O MIS, por outro lado, foi o mais demorado para aplicar, ao mesmo tempo em que foi o mais rico em detalhes. Entretanto, foi um desafio fazer a análise segmentando os três tipos de signos: metalinguísticos, estáticos e dinâmicos. A avaliação por observação forneceu percepções muito próximas de uma situação real de uso. Contudo, a interação com a participante voluntária exigiu rigor na condução do método para não influenciar na realização da tarefa. O tempo necessário para a avaliação por observação foi intermediário entre a AH e o MIS. No entanto, deve-se ressaltar que apenas uma pessoa foi observada. $\mathrm{O}$ tempo de aplicação e análise irá aumentar gradativamente à medida que a interação de mais pessoas com o sistema seja observada.

No contexto atual de um mundo em pandemia, o recrutamento de voluntários ficou limitado a colegas de forma remota e familiares de convívio diário. Na percepção do estudante, as avaliações realizadas de modo remoto são mais fáceis de serem conduzidas, entretanto, poderiam apresentar limitações no que pode ser extraído de informações a distância, como linguagem corporal e expressões. A respeito do tema da tarefa observada ser a morte, experiências pessoais do próprio estudante permitiram uma percepção adequada da sensibilidade do tema e a possibilidade do tema ativar gatilhos psicológicos. Assim sendo, o uso de linguagem compassiva se fez presente sempre que possível ao conversar com voluntários e colegas de turma.

Os três métodos de avaliação revelaram aspectos de IHC que podem ser melhorados, contribuindo para o desenvolvimento de novas soluções para o tema, seja no Facebook ou em outras redes sociais. A aplicação de cada um deles também foi importante para perceber um balanço entre tempo de aplicação, dados coletados e restrições de aplicação.

Do ponto de vista ético, é interessante que haja certa prioridade para aprimorar os pontos de melhoria identificados pela aplicação dos três métodos. A experiência dá indícios de que pode haver certa confusão na configuração de contato herdeiro. Apesar de ser possível que um familiar solicite a exclusão ou a possibilidade de gerenciamento da conta memorial [Viana et al., 2017], seria melhor se não houvesse dúvidas quanto à configuração bem-sucedida da ferramenta pelo próprio titular do perfil.

$\mathrm{Na}$ perspectiva da docente que conduziu a disciplina a experiência foi bem sucedida. Ressalta-se que a disciplina já está na terceira edição no mesmo formato e abordando o mesmo tema, sendo que a segunda edição também gerou inovações sobre legado digital em aplicações computacionais distintas das relatadas neste artigo. Dessa forma, como trabalhos futuros, pretende-se diversificar os métodos de avaliação e os sistemas avaliados. 


\section{Referências}

Barbosa, S. D. J.; Silva, B. S. da; Silveira, M. S.; Gasparini, I.; Darin, T.; Barbosa, G. D. J. (2021) Interação Humano-Computador e Experiência do Usuário. Autopublicação.

de Souza, C. S., Leitão, C. F., Prates, R.O., and da Silva, E. J. (2006) The semiotic inspection method. In: Proceedings of VII Brazilian symposium on Human factors in computing systems (IHC '06). Association for Computing Machinery, New York, NY, USA, 148-157. DOI:https://doi.org/10.1145/1298023.1298044

Nielsen, J. (1994) Enhancing the explanatory power of usability heuristics. In: Conference Companion on Human Factors in Computing Systems (CHI '94). Association for Computing Machinery, New York, NY, USA, 210. DOI:https://doi.org/10.1145/259963.260333

Oliveira, J. P. M.; Wagner, F. R. and Gasparini, I. (2020) A formação em computação. In: Cristiano Maciel; José Viterbo. (Org) Computação e sociedade: a profissão volume 1. [e-book]/ $1^{\text {a }}$ edição. Cuiabá-MT: EdUFMT Digital.

Pimentel, M and Carvalho, F. d. S. P. (2020) Princípios da Educação Online: para sua aula não ficar massiva nem maçante! SBC Horizontes, maio 2020. ISSN 2175-9235, http://horizontes.sbc.org.br/index.php/2020/05/23/principios-educacao-online.

Acesso em 20 de agosto de 2021.

Preece, J.; Sharp, H.; Rogers, Y. (2013) Design de Interação - além da interação humano-computador. Tradução: Isabela Gasparini; Revisão técnica: Marcelo Soares Pimenta. $3^{a}$ ed. Porto Alegre: Bookman.

Taylor, C. (2019) Facebook adds new tools for handling accounts of deceased users. https://www.cnbc.com/2019/04/10/facebook-adds-new-tools-for-handling-accounts-o f-deceased-users.html. Acesso em: 09 julho 2021.

Trevisan, D.; Maciel, C. (2020) Morte, Educação e Tecnologias Digitais: Reflexões em tempos de Pandemia. SBC Horizontes, jul. 2020. ISSN 2175-9235, http://horizontes.sbc.org.br/index.php/2020/07/02/morte-educacao-e-tecnologias-digi tais/. Acesso em: 20 agosto 2021.

Viana, G. T.; Maciel, C.; Arruda, N.; Souza, P. de. (2017) Análise dos Termos de Uso e Políticas de Privacidade de Redes Sociais quanto ao Tratamento da Morte de Usuários. In: VIII Workshop sobre Aspectos da Interação Humano-Computador para a Web Social, 2017, Joinville. Anais do VIII Workshop sobre Aspectos da Interação Humano-Computador na Web Social (WAIHCWS'17). [S.I.]:SBC, 2017. p. 82-93. ISSN: 1613-0073, http://ceur-ws.org/Vol-2039/paper08.pdf 